\title{
OBSERVATION OF WAVE SPECTRUM UNDER TYPHOON CONDITION
}

\author{
Woo Jeong Sim¹, Hyun-Doug Yoon², Weon Mu Jeong ${ }^{3}$ and Kyong-ho Ryu ${ }^{4}$
}

\begin{abstract}
The spatial and temporal variation of wave spectrum under typhoon SANBA, which was the $16^{\text {th }}$ typhoon originating in the northwestern Pacific Ocean in 2012, was examined. The typhoon SANBA passed through the middle of Korean peninsula whereas most of typhoons passed the Straits of Korea (eastern side of Korean peninsula). This unique path of typhoon SANBA provided interesting features of wave conditions. It was believed that wave heights were larger in the right side of the typhoon path because typhoons translation speed and rotational wind field were in the same direction (i.e., dangerous semicircle). However, observational evidence of wave spectrum under typhoon was still rare because of the difficulty in measurement. In this study, wave spectrum analysis under extreme storm condition of typhoon SANBA is given for the left and right sides of the path, including the information of significant wave height, peak period, and dominant wave direction. Wave measurements were obtained by directional wave rider buoys installed at Yeosu and Namhyeongjedo in Korea. Yeosu and Namhyeongjedo are located in each of the left and right of the path of the typhoon SANBA. The measurements provided distinguished wave conditions in each side of navigable semicircle and dangerous semicircle of the typhoon. Therefore it is possible to observe the spatial differences of the wave spectrum across the path of the typhoon, as well as temporal changes of wave spectrum as the typhoon evolves.
\end{abstract}

Keywords: Frequency spectrum, Directional Spectrum, Cross-spectrum, Typhoon SANBA

\section{INTRODUCTION}

Characteristic of waves generated by typhoon is different from those generated by wind. Since typhoon supplies continuous energy to waves while traveling in the same direction with waves, the energy of the low-frequency components of the wave spectrum increases rapidly. In general, the rapid increase of wave energy occurs 3-4 hours prior to the maximum wind speed. Because such increase in the low-frequency wave energy occurs strongly in a short time, the shape of wave spectrum during the passage of typhoon is very different from that produced by typical wind waves, showing a bimodal aspect.

Considering the importance of wave conditions for managing coastal area, it is vital to understand the characteristics of wave field, including frequency and wave direction spectrum, under typhoon condition. Kim (2012) measured wave conditions inside Busan harbour which was frequently affected by typhoons. It was found that the rapid change of wave direction during the typhoons passage caused large wave heights. Jeong et al. (2014) reported the effect of three typhoons in 2004 2006. They observed wave conditions at a number of observation locations during the passage of typhoons and contributed for validation of numerical models.

Especially, in the Northern Hemisphere, this phenomenon is more intense within the dangerous semicircle, the right hand segment of the typhoon where the typhoon's translation and rotational wind are additive. But within the navigable (safe) semicircle where the typhoon and rotational wind proceeds in the opposite direction, the damage from typhoon is always less than that in the dangerous semicircle. Because the wave energy becomes stronger in a very short period, the wave spectrum of typhoon is shaped differently from that of general wind waves. However, the information of the influence of typhoon on wave conditions is still rare because of its severe condition for maintaining measurement equipment.

The main purpose of this study is to examine the spatial and temporal changes of wave spectrum analysis (frequency, directional, cross-spectrum analysis) in accordance with the proceeding of Typhoon SANBA in 2012. It affected the Southern coastal area of South Korea accompanying strong winds with over $43.9 \mathrm{~m} / \mathrm{s}$ of maximum instantaneous wind speed and heavy rain of $863.5 \mathrm{mmh}$. The typhoon SANBA passed through in the middle of Korean peninsula whereas most of typhoons passed the Straits of Korea (eastern side of Korean peninsula). This unique path of typhoon SANBA provided interesting features of wave conditions.

\section{OBSERVATION}

The wave conditions were collected simulataneously at Yeosu (St.1) and Namhyeongjedo (St.2) by Korea Institute of Ocean Science \& Technology (KIOST). The locations of Yeosu and Namhyeongjedo

\footnotetext{
${ }^{1}$ Department of Harbor, Kunil Eng., Seoul, Republic of Korea

2 Department of Civil and Environmental Eng., Myongji University, Yongin, Republic of Korea

${ }^{3}$ Coastal Disaster Prevention Research Center, Ansan, Republic of Korea

${ }^{4}$ Coastal Disaster Prevention Research Center, Ansan, Republic of Korea
} 
are provided in Table 1. Also, Figure 1 shows the tracking path of Typhoon SANBA and the locations of the measurement sites. The intensity scale of typhoon SANBA can be categorized into TD (Tropical Depression), TS (Tropical Storm), STS (Severe Tropical Storm) and TY (Typhoon), based on sustained wind speeds. The ranges of maximum wind speed near the center of typhoon for each stage are less than $17 \mathrm{~m} / \mathrm{s}$ for TD, 17 24 m/s for TS, 25 32 m/s for STS, larger than $33 \mathrm{~m} / \mathrm{s}$ for TY. Yeosu and Namhyeongjedo are located in the left and right sides of the tracking path of the typhoon SANBA near Korean peninsula. Thus, the two regions are respectively represent the navigable semicircle and dangerous semicircle of the typoon.

\begin{tabular}{c|c|c|c}
\multicolumn{4}{c}{ Table 1. Measurement stations } \\
\hline \hline \multirow{2}{*}{ No. } & \multirow{2}{*}{ Station } & \multicolumn{2}{|c}{ Location } \\
\cline { 3 - 4 } & & Latitude & Longitude \\
\hline St.1 & Yeosu & $34^{\circ} 23^{\prime} 134^{\prime \prime}$ & $127^{\circ} 51^{\prime} 156^{\prime \prime}$ \\
\hline St.2 & Namhyeongjedo & $34^{\circ} 57^{\prime} 33.6^{\prime \prime}$ & $128^{\circ} 54^{\prime} 10.5^{\prime \prime}$ \\
\hline
\end{tabular}

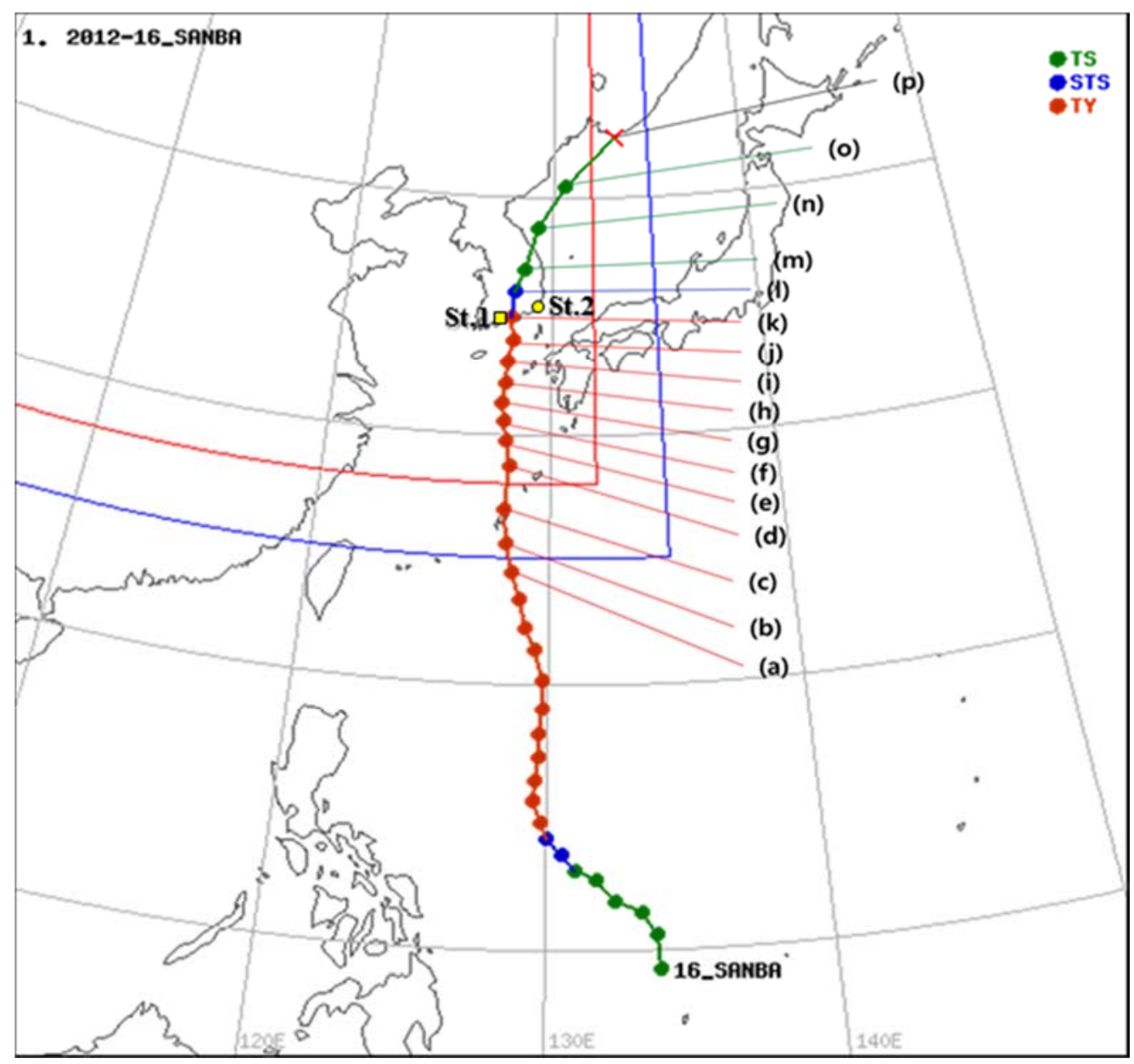

Figure 1. Location map of measurement stations and storm lane (modified from National Typhoon Center (typ.kma.go.kr))

Table 2 lists the time tables of the wave measurements. The spectral analysis was performed with the measurement data from 21:00 of Sep 15, 2012 to 09:00 of Sep 17, 2012. During this period, the spectral analysis was conducted for every 3 hours. The data were obtained using directional wave rider buoys, and they were recorded for 1600 s every 30 minutes and sampled at $1.28 \mathrm{~Hz}$. The buoy contains heave, pitch, and roll sensors, whose signals are converted to surface elevation and two horizontal velocity components for directional spectrum analysis. 
Table 2. Time table of measurement

\begin{tabular}{c|c|c|c}
\hline \hline No. & Date & No. & Date \\
\hline (a) & $2012-09-1521: 00$ & (i) & $2012-09-17$ 06:00 \\
\hline (b) & $2012-09-16$ 03:00 & (j) & $2012-09-17$ 09:30 \\
\hline (c) & $2012-09-16$ 09:00 & (k) & $2012-09-17$ 12:00 \\
\hline (d) & $2012-09-16$ 15:00 & (l) & $2012-09-17$ 15:00 \\
\hline (e) & $2012-09-1618: 00$ & (m) & $2012-09-17$ 18:00 \\
\hline (f) & $2012-09-1621: 00$ & (n) & $2012-09-1721: 00$ \\
\hline (g) & $2012-09-1700: 00$ & (o) & $2012-09-1703: 00$ \\
\hline (h) & $2012-09-1703: 00$ & (p) & $2012-09-1709: 00$ \\
\hline
\end{tabular}

\section{RESULTS AND DISCUSSION}

\section{Frequency Spectrum}

Figure 2 show the frequency spectrum at Yeosu (St.1) which is located in the left side of typhoon SANBA. The horizontal axis represents frequency $(f)$, and the vertical axis indicates the spectral density of the frequency $(S(f))$ with a unit of $\mathrm{m}^{2} / \mathrm{Hz}$. The solid red lines represent the smoothed wave spectrum with bin-averaging. In the early stages ((a)-(h)), the spectrum intensity was very small, being less than $1 \sim 3 \mathrm{~m}^{2} / \mathrm{Hz}$. When the typhoon SANBA passed through Korean Peninsula ((j)-(l)), the frequency spectrum showed a bi-modal aspect, with increasing of low-frequency components $(f=0.05 \sim 0.12 \mathrm{~Hz})$. The increase of low-frequency components was probably due to swell generated by the typhoon SANBA. In addition to the large low-frequency components, another spectral peak was also found around $f=0.17$ $\mathrm{Hz}$. This is a typical peak frequency range for wind-generated wave conditions. Overall, the spectrum shows a bi-modal shape, with a double peak in the low-frequency and wind-generated peak wave frequency.

As the typhoon SANBA moved away from Korean peninsula ((m)-(p)), the intensity of typhoon SANBA rapidly decreased, and the magnitude of the spectral density was also significantly decreased. Also, during this period, the two peaks of bi-modal shape were merged.

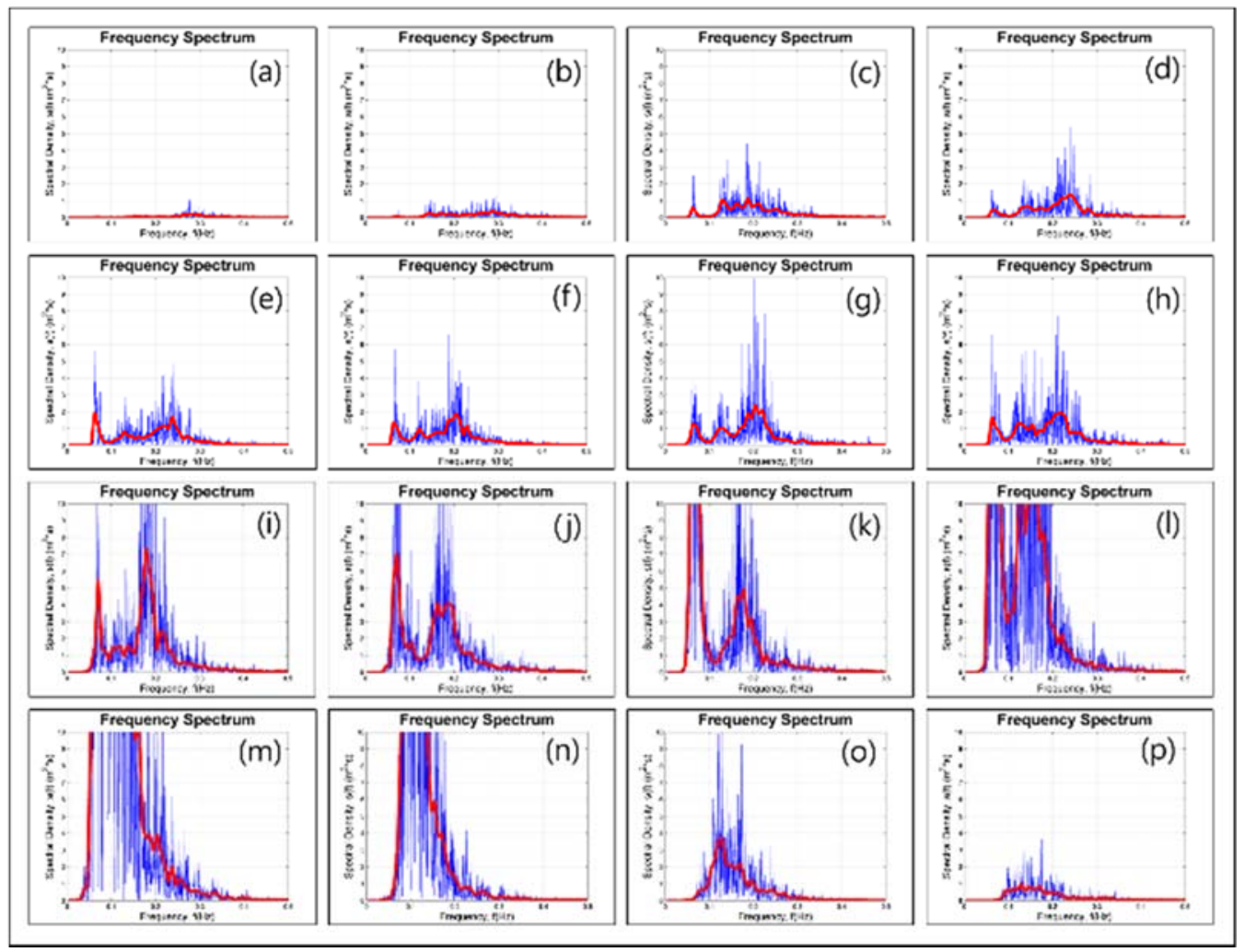

Figure 2. Frequency spectrum at Yeosu. 
Figure 3 show the frequency spectrum at Namhyeongjedo which is located in the right side of typhoon SANBA. General trend is similar to that of Yeosu except that there is a 3-hour time shift between the two locations. The peak intensity of the frequency spectrum at Namhyeongjedo occurred at time (n) approximately 3 hours later than Yeosu.

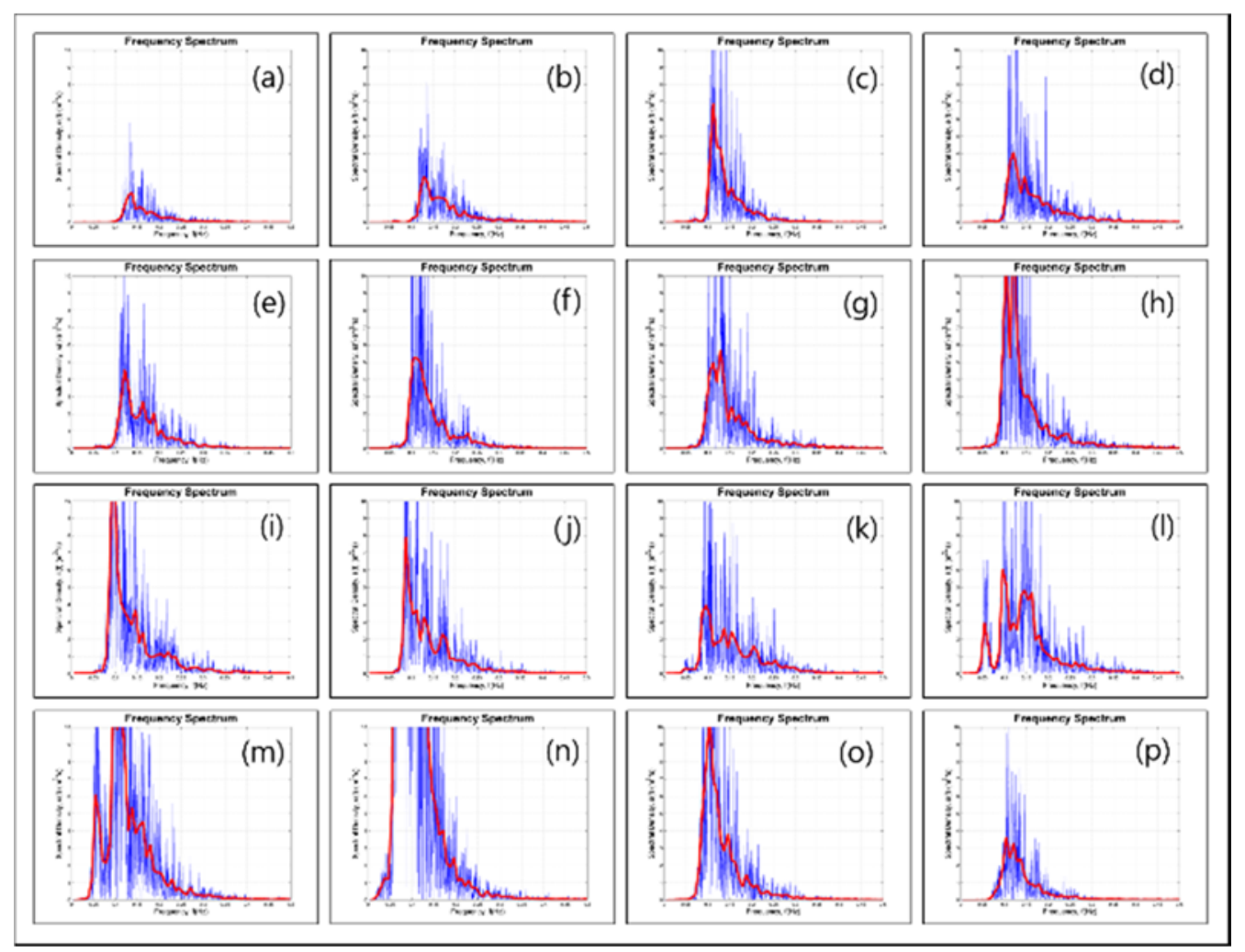

Figure 3. Frequency spectrum at Namhyeongjedo.

\section{Directional Spectrum}

Figures 4 and 5 show the directional spectrum at Yeosu and Namhyeongjedo, respectively. The directional spectrum was made using the Extended Maximum Entropy Method (EMEP) by Hashimoto et al. (1994) using 3 quantity point measurement such as surface elevation and two horizontal velocity components. The outward radial scale represents the increase of the wave frequency $(\mathrm{Hz})$. The red color means higher wave energy intensity. The angle of the circle indicates wave direction. Consistent to the frequency spectrum, the wave energy intensity also showed the maximum value at (m) at Yeosu and date (n) at Namhyeongjedo. The dominant directions of Yeosu and Namhyeongjedo were initially $140^{\circ} \sim 180^{\circ}$ which means that most of wave were coming from the south. However, the dominant directions were rapidly changed to approximately $300^{\circ}$ under severe typhoon conditions.

Table 3 summarizes significant wave height $\left(H_{s}\right)$, peak period $\left(T_{p}\right)$, and dominant wave direction $\left(D_{p}\right)$. Consistent to the frequency and directional wave spectrum, largest $H_{s}$ occurred during $(\mathrm{m})$ at Yeosu and during (n) at Namhyeongjedo. The largest wave height at Namhyeongjedo $\left(H_{s}=10.48 \mathrm{~m}\right)$, which located in the dangerous semi-circle, was larger than that at Yeosu $\left(H_{s}=9.96 \mathrm{~m}\right)$, although Namhyeongjedo is located farther from the typhoon path (Figure 1). The peak period corresponding to the largest significant wave height were $16.67 \mathrm{~s}$ and $14.29 \mathrm{~s}$, respectively, and this is consistent with that most of wave energy was located in the low-frequency components in Figures 2 and 3. Also, it is found that the dominant wave directions under typhoon SANBA were ENE, whereas the dominant wave direction was S when approaching to Korean peninsula. 


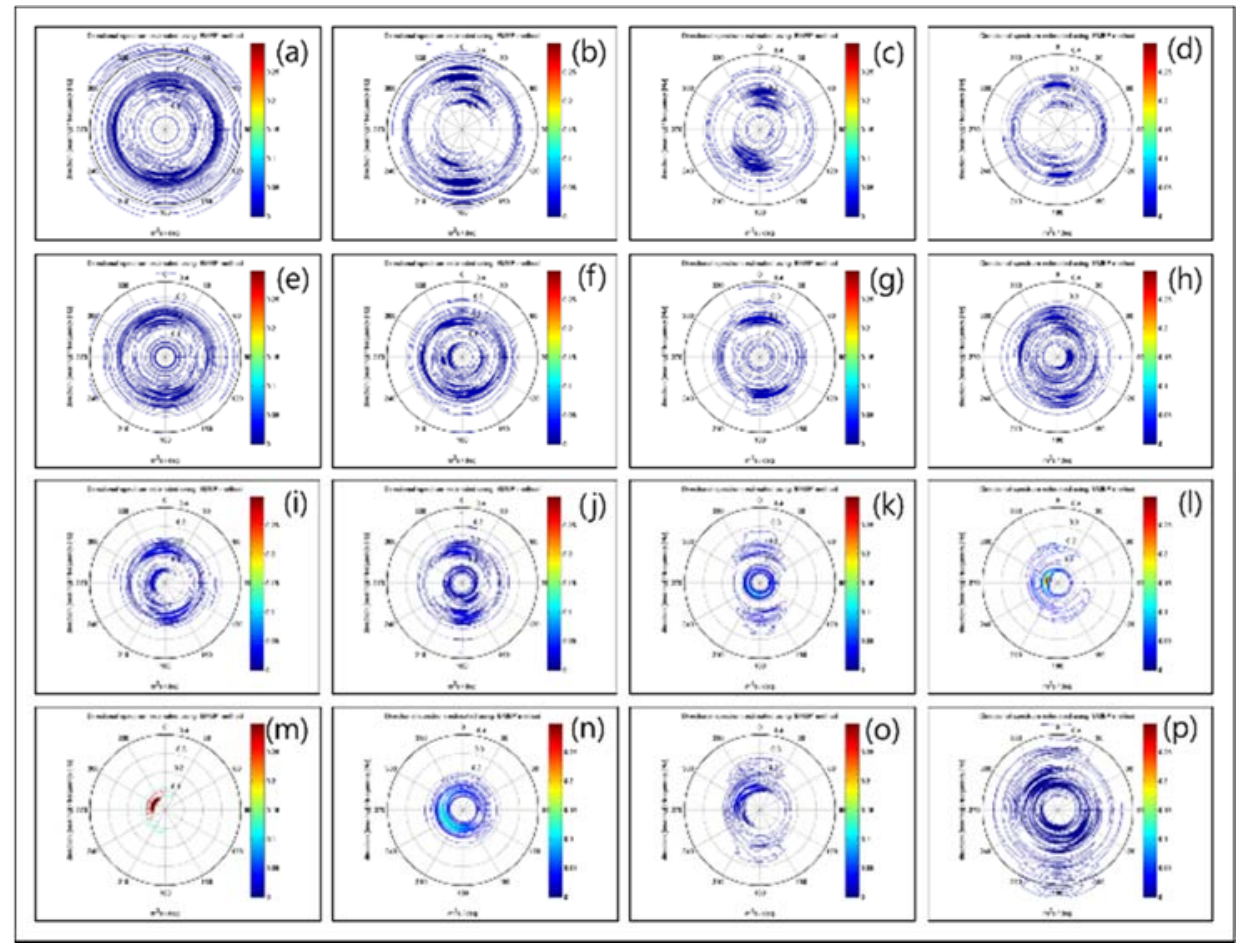

Figure 4. Directional spectrum at Yeosu

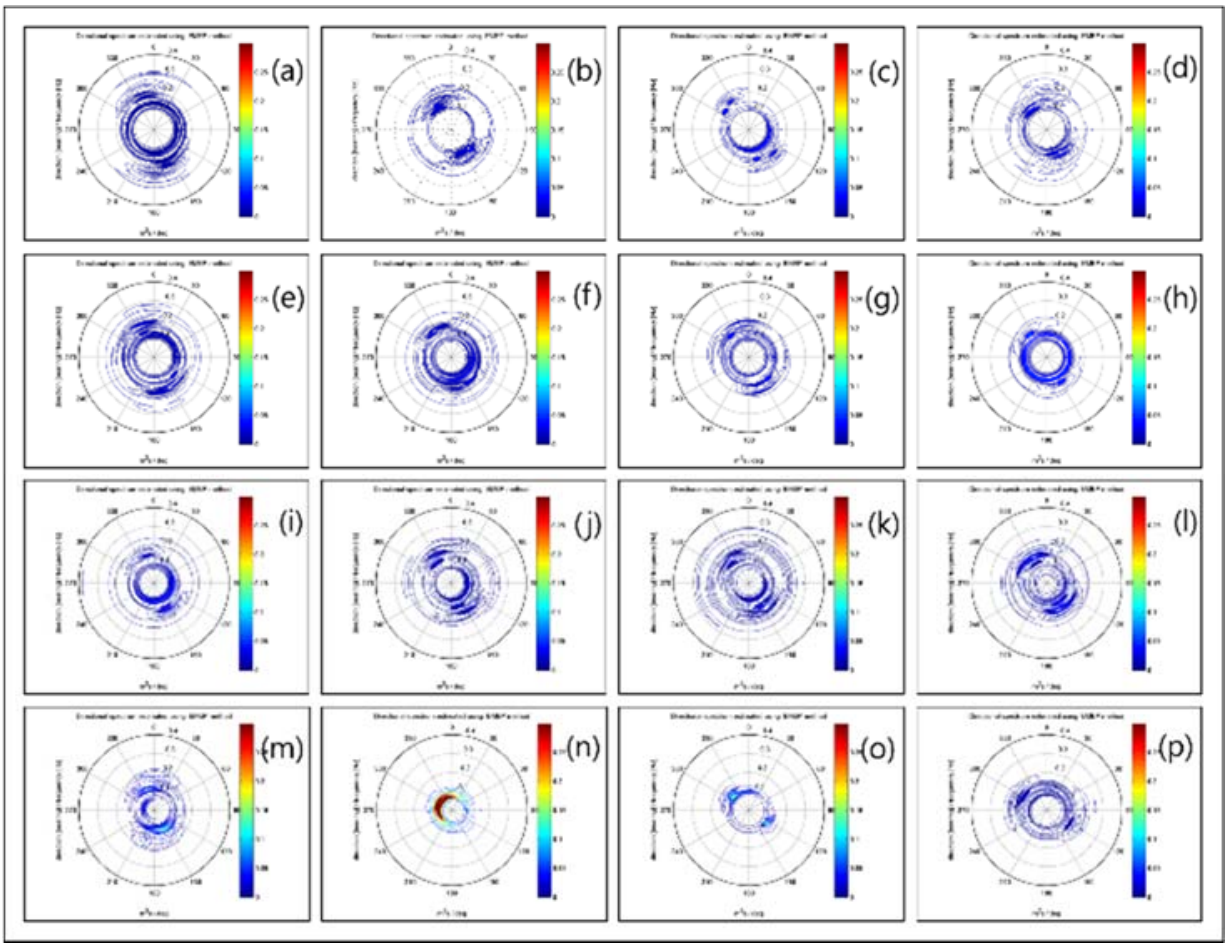

Figure 5. Directional spectrum at Namhyeongjedo 


\section{Cross-Spectrum}

Figure 6 shows the cross-spectrum between Yeosu and Namhyeongjedo. The cross power spectral density is the distribution of power per unit frequency and defined as:

$$
P_{x y}(\omega)=\sum_{m=-\infty}^{\infty} R_{x y}(m) e^{-i \omega m}
$$

where the cross-correlation sequency $R_{x y}(m)=E\left\{x_{n+m} y_{n}^{*}\right\}=E\left\{x_{n} y_{n-m}^{*}\right\}, x_{n}$ and $y_{n}$ are jointly stationary random processes, $n$ is an integer, and $E\{\cdot\}$ is the expected value operator. The horizontal scaling means frequency $(\mathrm{Hz})$ and the vertical scaling means the power of cross-spectrum.

Table 3. Measured significant wave height $\left(\mathrm{H}_{\mathrm{s}}\right)$, peak period $\left(T_{p}\right)$, dominant wave direction $\left(D_{p}\right)$

\begin{tabular}{|c|c|c|c|c|c|c|}
\hline \multirow[b]{2}{*}{ No. } & \multicolumn{3}{|c|}{ Yeosu } & \multicolumn{3}{|c|}{ Namhyeongjedo } \\
\hline & $\begin{array}{c}\mathrm{H}_{\mathrm{s}} \\
(\mathrm{m})\end{array}$ & $\begin{array}{l}T_{p} \\
(s)\end{array}$ & $\begin{array}{l}D_{p} \\
\left({ }^{\circ}\right)\end{array}$ & $\begin{array}{l}\mathrm{H}_{\mathrm{s}} \\
(\mathrm{m})\end{array}$ & $\begin{array}{l}\mathrm{T}_{\mathrm{p}} \\
(\mathrm{s})\end{array}$ & $\begin{array}{l}D_{p} \\
\left({ }^{\circ}\right)\end{array}$ \\
\hline (a) & 0.60 & 3.57 & 182 & 1.29 & 7.14 & 159 \\
\hline (b) & 0.99 & 3.45 & 184 & 1.66 & 7.69 & 328 \\
\hline (c) & 1.42 & 7.15 & 198 & 2.03 & 9.09 & 165 \\
\hline (d) & 1.56 & 4.17 & 178 & 1.96 & 7.69 & 151 \\
\hline (e) & 1.68 & 4.17 & 178 & 2.17 & 8.33 & 338 \\
\hline (f) & 1.81 & 5.00 & 176 & 2.43 & 8.33 & 143 \\
\hline (g) & 1.93 & 4.76 & 356 & 2.47 & 9.09 & 140 \\
\hline (h) & 2.03 & 4.35 & 170 & 3.40 & 8.33 & 150 \\
\hline (i) & 2.89 & 5.88 & 344 & 2.94 & 10.00 & 154 \\
\hline (j) & 2.88 & 14.29 & 178 & 2.37 & 11.11 & 159 \\
\hline (k) & 3.86 & 16.67 & 171 & 2.38 & 11.11 & 148 \\
\hline$(\mathrm{I})$ & 5.21 & 16.67 & 269 & 2.79 & 6.25 & 148 \\
\hline (m) & 9.96 & 16.67 & 299 & 3.73 & 9.09 & 156 \\
\hline (n) & 5.00 & 12.50 & 228 & 10.48 & 14.29 & 299 \\
\hline (o) & 2.20 & 8.33 & 340 & 2.99 & 10.00 & 312 \\
\hline (p) & 1.33 & 6.25 & 180 & 1.91 & 9.09 & 303 \\
\hline
\end{tabular}

The cross-spectrum between Yeosu and Namhyeongjedo shows the largest value at $(\mathrm{k})$, when the typhoon SANBA just landed on Korean Peninsula. After the typhoon SANBA lands on Korean Peninsula, the cross-spectrum decreased rapidly. Also, the cross-spectrum showed the largest value in the lowfrequency range $(f=0.057 \mathrm{~Hz})$. This implies that low-frequency coherent components (i.e., swell) dominates the wave condition in both Yeosu and Namhyeongjedo under typhoon condition rather than wind waves components. 


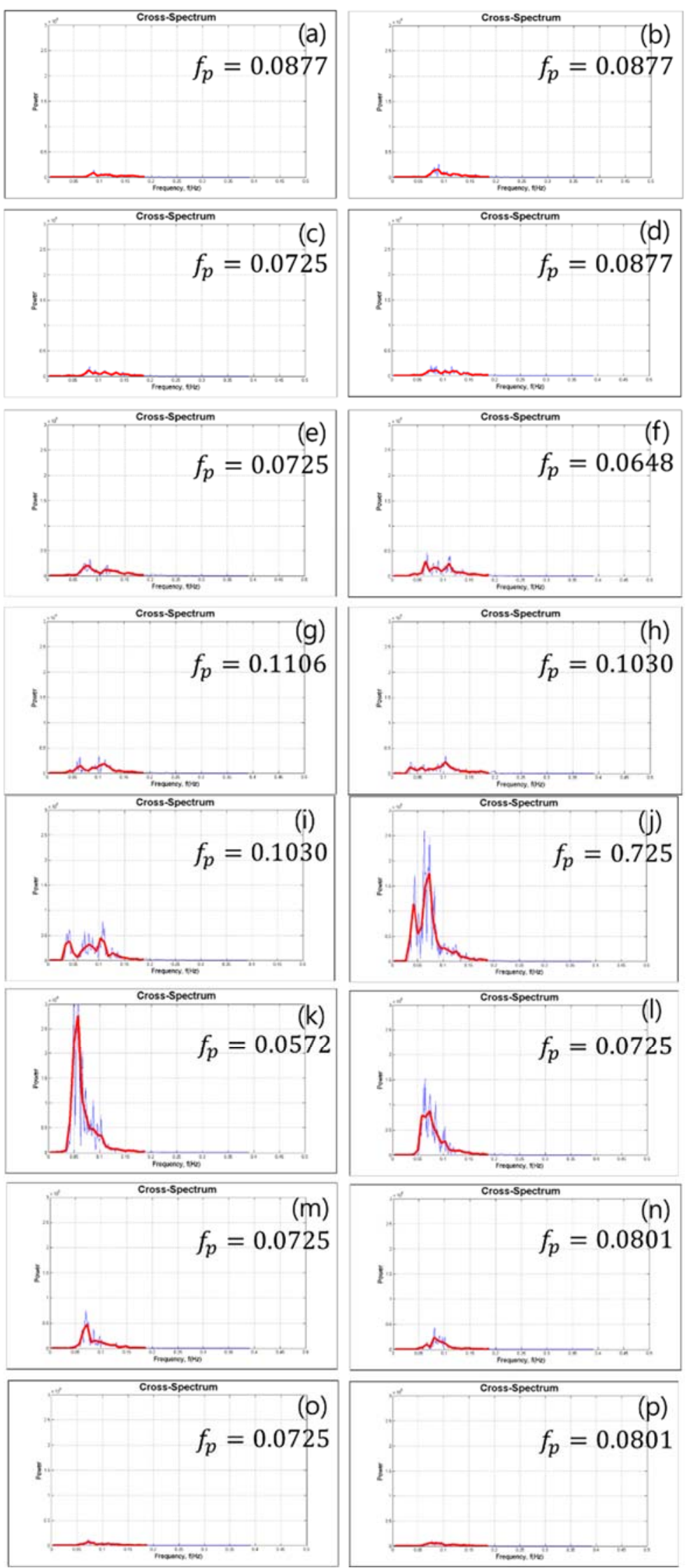

Figure 6. Cross-spectrum between Yeosu and Namhyeongjedo 


\section{CONCLUSIONS}

The spatial and temporal variation of wave spectrum under typhoon SANBA was examined at Yeosu and Namhyeongjedo which were located in the left (navigable semicircle) and right (dangerous semicircle) sides of the typhoon path. The frequency spectrum shows a bi-modal aspect for both Yeosu and Namhyeongjedo but there was a 3-hour difference of the peak wave energy. The directional spectrum shows that the dominant wave direction was rapidly changed under severe typhoon conditions. Also, the significant wave height at Namhyeongjedo (dangerous semi-circle) was larger than that at Yeosu (navigable semi-sircle), supporting the argument that wave condition is severe in the dangerous semicircle. The peak period corresponding to the largest significant wave heights were approximately 15 s due to the swell generated by the typhoon. Finally, the cross-spectrum analysis showed that the frequency spectrum between Yeosu and Namhyeongjedo were mostly cohererent in the low-frequency components, supporting that wave conditions under severe wave conditions were dominated by the lowfrequency motion (i.e., swell) rather than wind waves.

\section{ACKNOWLEDGEMENT}

This work was supported by Basic Science Research Program through the National Research Foundation of Korea (NRF) funded by the Ministry of Science, ICT \& Future Planning (2014R1A1A1008095) and Development of Fundamental Technology for Coastal Erosion Control (PE99523) funded by KIOST.

\section{REFERENCES}

Hashimoto, N., Nagai, T., and Asai., T., 1994. Extension of the maximum entropy principle method for directional wave spectrum estimation. Proc. 24th ICCE. Kobe, 1, pp 232-246.

Jeong, W.-M., Kim, S., Baek W. and Oh, S., 2014. Measurements of storm waves generated by typhoon passed through Eastside of Korea Strait from 2004 to 2006. Journal of Korean Society of Coastal and Ocean Engineers, 26(2), pp. 65-71.

Kim, D., 2012. Wave characteristics due to the path of Typhoon in Suyeong Bay. Pukyung Nat. Univ. (in Korean) 